\title{
Central Sensitization Mechanism in Chronic Knee Osteoarthritis
}

\section{EF Turovskaya $^{1,2}$, LI Alekseeva ${ }^{1 *}$, EG Filatova $^{2}$, EL Nasonov $^{1}$ and Cara Chimienti ${ }^{3}$}

${ }^{1}$ Federal State Budgetary Institution "V.A. Nasonova Research Institute of

Rheumatology", Moscow, Russian Federation

${ }^{2}$ I.M. Sechenov First Moscow State Medical University, Moscow, Russian Federation

${ }^{3}$ Registered Kinesiologist Tel., Canada

*Corresponding Author: LI Alekseeva, Federal State Budgetary Institution "V.A. Nasonova Research Institute of Rheumatology", Moscow, Russian Federation.
Received: November 17, 2021

Published: December 23, 2021

(C) All rights are reserved by LI Alekseeva

., et al.

\section{Abstract}

Pain is the main symptom of osteoarthritis (OA). Currently, there are few mechanism-based treatments that provide adequate and satisfactory pain control. Hence, there is a need to investigate pain mechanisms in OA.

Objective: To assess key mechanisms for pain in chronic OA.

Subjects and Method: 89 women aged 45-65 years with chronic knee OA pain were included in a prospective observational study. The study included clinical rheumatologic and neurological examinations, screening neuropathic pain scales (DN4 and Pain DETECT). Functional status and disability was assessed by WOMAC scale. Emotional disturbances (depression and anxiety) were examined by Hospital Anxiety and Depression scale. Knee joint instrumental examination involved radiographic and ultrasound studies.

Results: Neuropathic pain scales data demonstrated neuropathic descriptors present in OA patients such as: an electric shock, burning, numbness, and pins and needles. Neurological examination revealed no somatosensory deficit. Examination of the sensitive sphere indicated hyperalgesia in $53.7 \%$ of patients with $\mathrm{OA}$.

Patients were divided in accordance with presence or absence of secondary hyperalgesia ( $41.5 \%$ and $58.5 \%$ ). The presence of secondary hyperalgesia was characterized by more pronounced pain on VAS $-5.9 \pm 1.5 \mathrm{~cm}(\mathrm{p}=0.013)$, a significantly higher level of depression - $8.9 \pm 2.9$ ( $\mathrm{p}=0.003$ ), and more pronounced disturbance of joint function (WOMAC $1184 \pm 365 \mathrm{~mm}(\mathrm{p}=0.009)$ ). Examination of mood also revealed an increased level of anxiety in patients with secondary hyperalgesia $-8.9 \pm 2.9$, but no significant differences were recorded $(p=0.082)$. Secondary hyperalgesia was not associated with structural changes in a joint.

Conclusion: Our findings have demonstrated that in the pathogenesis of chronic pain in knee OA, two mechanisms take place: nociceptive and central sensitization. In one-third of patients central sensitization is predominant and determines the neuropathic pattern of pain. CS is characterized by the absence of neurological deficit and absence of pain intensity associated with structural changes in a joint. Therefore, one of the main ways of controlling pain should also target CNS mechanisms, including anticonvulsant, and antidepressant agents.

Keywords: Osteoarthritis; Chronic Pain; Neuropathic Descriptors; Central Sensitization

\section{Introduction}

Osteoarthritis $(\mathrm{OA})$ is the most common rheumatological disease worldwide [1]. The prevalence of OA is 8.2 cases per 100,
000 people; this increases with age, affecting $50 \%$ of those over 65 years old. In $10 \%$ of all cases, OA eventually leads to (significant) disability [2]. 
$\mathrm{OA}$ is the most common reason of pain in the elderly population [3], and a major cause of chronic pain in the general population (34\%), overtaking low back pain (15\%), trauma and surgery (15\%), headache (8\%) and other types of arthritis (8\%) [4]. Currently, pain control in patients with $\mathrm{OA}$ cannot provide adequate pain relief [5,6]. Recent European studies demonstrated low efficacy of NSAIDs in controlling OA pain ranging from $27 \%$ to $61 \%$ [4].

Traditionally, chronic pain in $\mathrm{OA}$ is considered to be a classical model of nociceptive pain, caused by structural and biochemical changes in bone, synovium and soft tissues [2].

But this model cannot explain certain dissociations revealed in a recent investigation dedicated to chronic pain in OA. First, the level of pain does not correlate with structural changes in knee OA [1]: up to $40 \%$ of individuals with radiological damage and soft tissue changes have no pain [2]. $44 \%$ of patients continue to suffer from persistent pain after total knee replacement [7].

Nociceptive mechanism of chronic pain in OA cannot explain the presence of clinical features: referred pain, secondary hyperalgesia (a phenomena of increased sensitivity to nociceptive stimuli at sites distant to the primary injury) $[8,9]$ and other sensitive phenomena which can occur in an intact region [10]. This data demonstrate that chronic pain in knee OA can also be caused by changes in CNS [2].

Hence, there is a need to investigate pain mechanisms in OA. Detailed pathogenic mechanism analysis may lead to the development of successful pain management based on mechanism- oriented treatment.

At the moment there are few studies dedicated to neurogenic mechanism in pain $\mathrm{OA}$.

Jacqueline R. Hochman., et al. in their focus group study on the hip/knee OA pain experience $(n=80)$ identified that participants used a broad range of descriptors that are suggestive of underlying neuropathic pain: for example, spontaneous paroxysms of pain, including electric shock-like sensations, burning, prickling, itching, heat, cold, pins and needles, numbness, tingling, and sensitivity to heat, cold, touch, or pressure. To distinguish nociceptive pain from neuropathic pain, 5 existing and validated questionnaires were completed by participants; the Pain DETECT, the Leeds Assessment of Neuropathic Symptoms and Signs pain scale with a self-report version, the Neuropathic Pain Diagnostic Questionnaire, ID Pain, and the Neuropathic Pain Questionnaire. 34\% of participants used neuropathic descriptors to characterize their pain. These participants were younger (mean \pm SD age $64.8 \pm 9.7$ years versus 72.0 \pm 10.0 years; $\mathrm{P}=0.003$ ), had a longer mean duration of $\mathrm{OA}$, higher pain intensity, and greater WOMAC severity, and were more likely to be women than those who did not use neuropathic descriptors, although only the age difference reached statistical significance [11].

Later, J.R. Hochman., et al. assessed the relationship between modified pain DETECT scores (mPD-Q) and signs of central sensitization on quantitative sensory testing (QST) in 36 chronic, symptomatic, knee OA individuals. QST signs of CS were defined as: mechanical hyperalgesia and/or enhanced temporal summation and/or allodynia. The relationship was assessed by such design: the presence of CS (yes/no) and a pre-selected mPD-Q score $(<12$ or $>12$ ). Among 57 eligible case knees, $45.6 \%$ had $\geq 1$ sign of $C S$. Controlling for age, knees with higher mPD-Q scores $(>12.0)$ had higher odds of having QST signs of CS (adjusted odds ratio (OR) =5.6; 95\% confidence interval (CI), 1.3-22.9). Thus, the mPD-Q may aid the identification of CS in people with chronic knee OA [12].

Gwilym., et al. in their study showed how and where central pain processing occurs in the human brain. For this aim they observed twenty patients with hip OA awaiting joint replacement and displaying signs of referred pain and ten age-matched controls. Patients were found to have significantly lower threshold perception to punctate stimuli by QST. They were also hyperalgesic to the noxious punctate stimulus in their areas of referred pain. To identify the supraspinal influences that underlie these clinical manifestations that the authors consider indicative of possible central sensitization, they used magnetic resonance imaging (MRI). Functional brain imaging illustrated significantly greater activation in the brainstem of OA patients in response to punctate stimulation of their referred pain areas compared with healthy controls, and the magnitude of this activation positively correlated with the extent of neuropathic-like elements to the patient's pain, as indicated by the Pain DETECT score. The authors concluded that periaqueductal grey activity is related to the clinical manifestations of disease rather than the presence of disease alone and is involved in the neuroplasticity associated with central sensitization in osteoarthritic pain [10]. 
There are several studies that try to answer the question: What kind of mechanisms in OA lead to chronic pain? Osteoarthritis is the most common rheumatological disease world and pain is its key symptom [13]. There is an acute need for effective pain control - efficacy of NSAIDs in controlling OA pain ranges from $27 \%$ to $61 \%$ [4]. Only a detailed understanding of chronic knee pain mechanisms can help control OA pain. Now it is clear that besides nociceptive pain there is another mechanism that takes place in chronic pain OA [14-16]. It can be reached only by a complex approach in examining patients with chronic knee OA that includes not only a rheumatological examination, but examination of the neurological sphere - especially sensitivity changes and emotional response to chronic pain.

In our study we tried in detail to examine knee pain mechanisms using rheumatological approach, neuropathic scales, neuropathic examination, emotional sphere and even algometry. As a result, our study can demonstrate the contribution of each mechanism and demonstrate what kind of medicaments are useful for manage chronic pain in OA.

However, there are several limitations in the existing studies.

As the first Jacqueline R. Hochman study [11] was designed to qualitatively assess the OA pain experience, there is no proportion of people with symptoms of neuropathic pain (quantitative study). The study has descriptive character, where validated questionnaires were used to reveal signs of neuropathic pain, but does not show whether neuropathic tests were positive and does not include neurological examination. According to the study, we can suppose that certain neurogenic mechanisms can take place in chronic knee pain, but we are unable to answer whether it is a neuropathic mechanism or central sensitization. Another question that the study does not answer is what the role of the neurogenic descriptors is. Second, information was not obtained on comorbid medical or neurologic conditions that may contribute to neuropathic symptoms, although people with other chronic pain conditions were excluded. Third, the small sample size limited the study's power to detect significant differences between participants who did and did not use pain descriptors suggestive of NP [11].

The limitations of the second Jacqueline R. Hochman study [12] are: defining central sensitization based on QST findings that have been linked with central sensitization in human experimental stud- ies [17], a limited number of study participants, and relying on a small group of controls to obtain reference QST values. So it is possible that sub-clinical sensory abnormalities went undetected in control knees, skewing reference data [12].

The limitations of the Gwilym., et al. study [10] are: the use of expensive and laborious methods (functional MRI and a limit number of study participants) [10].

As there is currently no "gold standard" for the diagnosis or evaluation of central sensitization [17] we include identification of central sensitization by several methods (neuropathic pain scales, but also investigation of pain system with the help of algometry, neurological and psychological examination). Such complex approach can help us also to reveal central sensitization in chronic knee pain on a subclinical level; to reveal association with pain intensity, joint function and structural changes, but also demonstrate the most convenient way of identifying central sensitization. The other advantage of the study is that we exclude individuals with other chronic pain, neurological disorders and psychosocial factors.

\section{Methods}

The clinical study protocol was approved by Interuniversity Medical and Pharmaceutical Ethic committee l and was performed in accordance with ethical standards, developed in line with the ethical standards of the Declaration of Helsinki. All patients provided written informed consent prior to embarking any study activities or procedures.

Since 2012 till 201389 consecutive women with symptomatic chronic knee $\mathrm{OA}$ and chronic pain were recruited and observed at the V.A. Nasonova Rheumatology Institute, Moscow. The study included females aged 45-65 years, who met the American College of Rheumatology clinical and radiographic criteria for the diagnosis of osteoarthritis of the knee and who had suffered pain for more than 3 months (criteria of chronic pain).

Exclusion criteria included: subjects aged $<45$ and $>65$ years; a (confounding) painful condition that was not connected with OA; patients who had psychiatric disorders that could compromise participation in the study; patients who were taking antidepressants, anticonvulsants and other drugs, that could influence the pain syndrome. 
Study design

The study included the examination of patients with knee OA at V.A. Nasonova Rheumatology Institute for study entry eligibility.

All eligible patients were observed by means of symptom and anamnesis, neurological examination with the accent on the sensitive sphere, rheumatological examination. Assessment of pain included analyzing its character, intensity and localization. Quality of life was assessed by European Quality of Life Questionnaire (EQ-5D). For examination of emotional disturbances, the hospital Anxiety and Depression Scale (HADs) scale was used. The scale was designed by Zigmond A.S. and Snaith R.P. in 1983 with the aim to reveal and assess depression and anxiety in general medical practice. The advantages of the scale are its ease of use and interpretation. That's why HADs can be used as a screening scale for emotional disturbance. The HADs scale contains 14 questions: 7 questions are screening tool for anxiety and other 7 are for depression. Depression subscale questions are based on more common complaints and symptoms typical for depression. Anxiety subscale questions based on standard clinical Present State Examination interview and private clinical experiment. Each question has four variant of answers. Only one answer can be chosen. Each answer can be evaluated from 0 to 3 , that reflect severity of emotional disorders. The higher subscale depression/anxiety is 21. 0-7 score means absence of anxiety/depression, 8-10 score - subclinical anxiety/depression, 11-15 score moderated signs of anxiety/depression, 16-21 score significant anxiety/depression syndrome [18].

Central sensitization was assessed by neuropathic pain scales: DN4; Pain DETECT $[19,20]$. The scales have been developed and validated for the purpose of identifying neuropathic elements of a patient's pain $[20,21]$. The result is a composite score ranging from 0 to 39 , where higher scores are more suggestive of neuropathic pain and lower scores are indicative of the pain being nociceptive. After neurological examination, that revealed secondary hyperalgesia (increased sensitivity to pain in intact region), the patients were categorized into two groups: I group with secondary hyperalgesia and II group without secondary hyperalgesia.

Secondary hyperalgesia is considered to be the clinical feature of central sensitization. Central sensitization was detected by pain sensation exam/explore with a needle that indicated hyperalgesia. Punctate stimuli were applied to control region(dorsal hand) with physiological stimuli and to low extremities using needle. In- creased sensitivity to pain to punctate stimulation or hyperalgesia was revealed in the damaged joint - primary hyperalgesia and increased sensitivity to pain in the intact region shin and even hip - secondary hyperalgesia.

Secondary hyperalgesia is considered to be a clinical feature of central sensitization (CS) [13]. The literature relating to models of musculoskeletal pain suggest that $\mathrm{OA}$ is associated with enhanced nociceptive transmission at the dorsal horn $[14,15]$ a hallmark of secondary hyperalgesia. This enhanced excitability of dorsal horn neurons to nociceptive inputs is termed central sensitization. This is manifested by 1) increased response to input from an injured or inflamed region, 2) increased response from regions adjacent to or remote from the injured/inflamed region, and 3) expansion of the receptive field of the spinal cord neuron $[16,17]$.

Primary hyperalgesia associates only with the damaged region. The key mechanism of primary hyperalgesia is peripheral sensitization or sensitization of nociceptors and increased response to mechanical and thermal stimuli only from the injured region [17].

\section{Statistical methods}

Focus groups (1- group with secondary hyperalgesia and 2group without secondary hyperalgesia) were compared. The parameters that were compared were; BMI, pain intensity, level of neuropathic tests, emotional disorders, quality of life, ultrasound, $\mathrm{X}$-ray, and level of functional activity of the knee joint. Descriptive statistics were calculated for all quantitative and qualitative variables. Student's t-tests were used for a normal distribution for all studied variables. Categorical variables were assessed using the Chi-square test. Significance was based on two-tailed tests with a $5 \%$ level of significance.

All analyses were performed using SPSS 16.0 software.

\section{Results}

- $\quad$ The mean age of subjects was $58.4 \pm 5.4$ years. The duration of $\mathrm{OA}$ since onset ranged from 3 years to 36 years. The Mean value of BMI was $33.11 \pm 7 \mathrm{~kg} / \mathrm{m}^{2}$ (obesity I-II stage).

- $\quad$ Significantly more patients had Kellgren-Lawrence grade II (75\%), several grade III grade $(20 \%)$ and only a few had grades I and IV.

- Mean value of pain insensitivity was $5.2 \pm 2 \mathrm{~cm}$. Mean value of knee WOMAC was $1049 \pm 439 \mathrm{~mm}$ (range from 0 to 2300 $\mathrm{mm}$ ) (Table 1). 


\begin{tabular}{|l|c|}
\hline Parameters & Value \pm SD \\
\hline Mean age, years & $58 \pm 5.4$ \\
\hline Duration of illness, years & $110 \pm 77$ \\
\hline BMI kg/m ${ }^{2}$ & $33.11 \pm 7$ \\
\hline $\begin{array}{l}\text { Kellgren-Lawrence grade, } \\
\text { n(\%) }\end{array}$ & \\
\hline I & $2(3.3 \%)$ \\
\hline II & $45(75 \%)$ \\
\hline III & $12(20 \%)$ \\
\hline IV & $1(1.7 \%)$ \\
\hline Pain intensity, VAS, (cm) & $5.2 \pm 2$ \\
\hline WOMAC, mm & $1049 \pm 439$ \\
\hline
\end{tabular}

Table 1: Characteristics of participants.

Pain syndrome analysis by neuropathic DN4 and Pain DETECT scales.

Neuropathic DN4 pain scale data demonstrated a median score of $3 \pm 1.76$. Scores $<4$ suggest neuropathic pain is unlikely, $\geq 4$ indicate a possible neuropathic component, and $37 \%$ patients had a DN4 score $\geq 4$

Both groups of patients with DN4 $\geq 4$ and DN4 $<4$ had neuropathic descriptors.

The frequency of neuropathic descriptors in patients with OA is represented in the most common descriptors were: resembling an electric shock- 59.6\%, burning - 47.2\%, pins and needles- $46.1 \%$, numbness - 42.7\%. 28.1\% experienced chills, 18\% mechanical hypoesthesia, $18 \%$ allodynia, $16.9 \%$ - cold sensitivity, $16.9 \%$ decreased sensitivity to needles and $10.1 \%$ had an itching sensation.

Patients with DN4 $\geq 4$ reported neuropathic descriptors more often than those with DN4<4 with the exception of burning and itching (Table 2).

\begin{tabular}{|c|c|c|c|}
\hline OA Pain characteristics & DN4 $\geq 4(33)$ & $\begin{array}{c}\text { DN4<4 } \\
\text { (56) }\end{array}$ & Value $p$ \\
\hline Burning, $\%$ & 51.5 & 44.6 & 0.530 \\
\hline Cold sensitivity, $\%$ & 30.3 & 8.9 & 0.009 \\
\hline Electric shock-like stimuli,\% & 87.9 & 42.9 & 0.000 \\
\hline Chills,\% & 60.6 & 8.9 & 0.000 \\
\hline Pins and needles, $\%$ & 60.6 & 37.5 & 0.034 \\
\hline Numbness, $\%$ & 75.8 & 23.2 & 0.000 \\
\hline Itching & 18.2 & 5.4 & 0.053 \\
\hline
\end{tabular}

\begin{tabular}{|l|c|c|c|}
\hline mechanical hypoesthesia & 33.3 & 8.9 & 0.004 \\
\hline $\begin{array}{l}\text { decreased sensitivity to } \\
\text { needles }\end{array}$ & 27.3 & 10.7 & 0.044 \\
\hline Allodynia & 36.4 & 7.1 & 0.001 \\
\hline
\end{tabular}

Table 2: Frequency of neuropathic descriptors in patients with DN $4<4$ and DN4 $\geq 4$.

The most common sensitive phenomena in patients with DN4 $\geq 4$ were electric shock- like stimuli, chills, pins and needles and numbness (Figure 1).

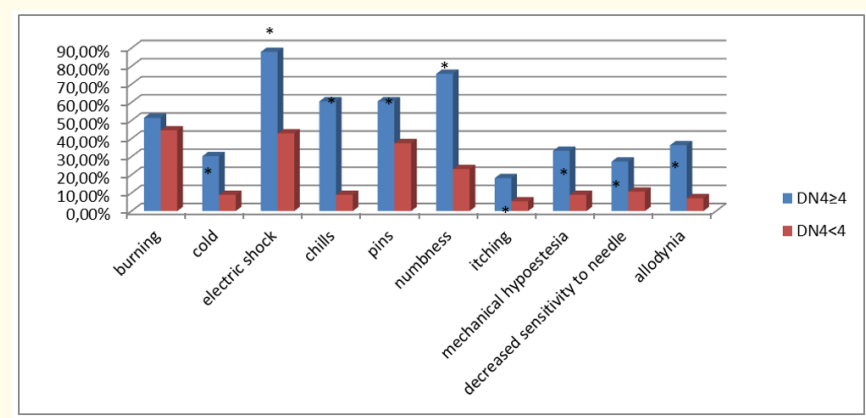

Figure 1: The frequency of neuropathic descriptors in patients with OA.

Central sensitization was also revealed by Pain DETECT score. Statistical data produced a median score of $13.6 \pm 6.6$ (maximum 38 scores). $25.6 \%$ had a definite neuropathic component (score range 19-38), 31.1\% had a possible neuropathic component (score range $13-18$ ), and in $43.3 \%$ (score range $0-12$ ), neuropathic pain was unlikely (Table 3 ).

\begin{tabular}{|l|c|}
\hline Pain DETECT value & $\mathbf{N}=\mathbf{8 9}$ \\
\hline Unlikely neuropathic pain, score range 0-12 & $38(42.7 \%)$ \\
\hline Possible neuropathic pain, score range 13-18 & $28(31.5 \%)$ \\
\hline Definite neuropathic pain, score range 19-38 & $23(25.8 \%)$ \\
\hline
\end{tabular}

Table 3: Neuropathic component detected by Pain DETECT scale.

The neuropathic pain scale data has demonstrated that patients with knee OA use descriptors typical for neuropathic pain. Neuropathic scale screening allows the mixed nature of chronic pain in $\mathrm{OA}$ to be revealed: besides nociceptive mechanisms, neurogenic mechanisms can occur. 
Specificity of neurological status in patients with $\mathrm{OA}$

When examining neurological status, emphasis on the peripheral nervous system was done.

Neurological examination did not reveal any deficit in either group. But in the sensitive sphere certain changes were revealed: hyperalgesia, numbness and even allodynia. In patients with DN4 $\geq 4$, changes in the sensitive sphere were statistically more common compared with patients with DN4<4 76.2\% (16) vs $50 \%$ (19), $p=0.046$. In patients with $\mathrm{DN} \geq 4$, the numbness of low extremities at different locations occurred in $16.1 \%$ (5) vs. $3.9 \%$ (2) of patients with $\mathrm{DN} 4<4(\mathrm{p}=0.055)$.

The changes in the sensitive sphere in patients with OA were more often accompanied by hyperalgesia (increased sensitivity to pain stimuli) and occurred in $53.7 \%$ patients. Hyperalgesia occurred in both groups of patients with DN4 $\geq 451.5 \%$, and in patients with DN4<4 49.1\%, no statistically significant difference was found, $p=0.826$. Hyperalgesia can be subdivided into primary hyperalgesia (increased sensitivity to pain in the damaged joint) and secondary hyperalgesia (increased sensitivity to pain in the intact region shin and even hip). Patients with DN $4 \geq 4$ had secondary hyperalgesia more often than patients with DN $4<4,58.5 \%$ vs $41.5 \%$, but the difference was not statistically significant $(p=0.270)$.

Localization of secondary hyperalgesia varies: hyperalgesia from the upper third of the shin to the lower third of the shin (25.7\%); from the lower third of the hip till the foot (22.6\%); from the knee till the lower third of the shin (19.4\%), hyperalgesia of lower third (19.4\%), hyperalgesia of upper third and middle third of the shin (6.5\%), hyperalgesia of upper third of the shin $(3.2 \%)$, hyperalgesia of middle shin till foot (3.2\%) (Figure 2,3).

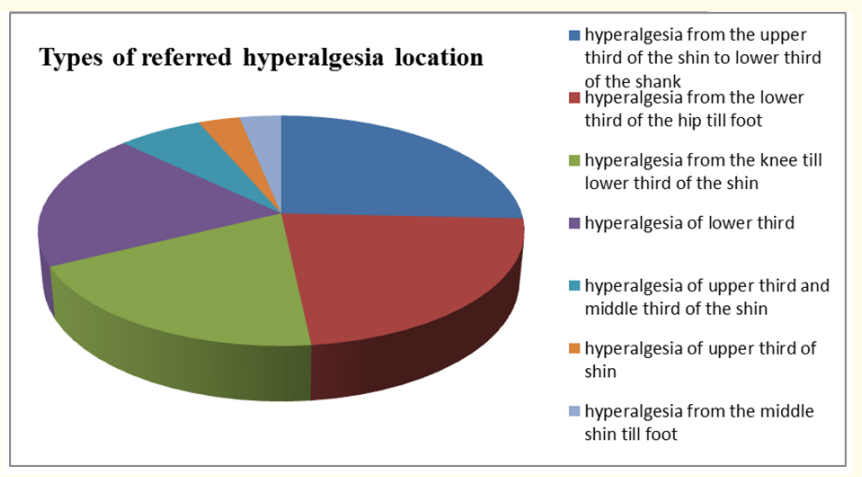

Figure 2: Frequency of neuropathic descriptors in patients with DN4 $<4$ and DN4 $\geq 4$.

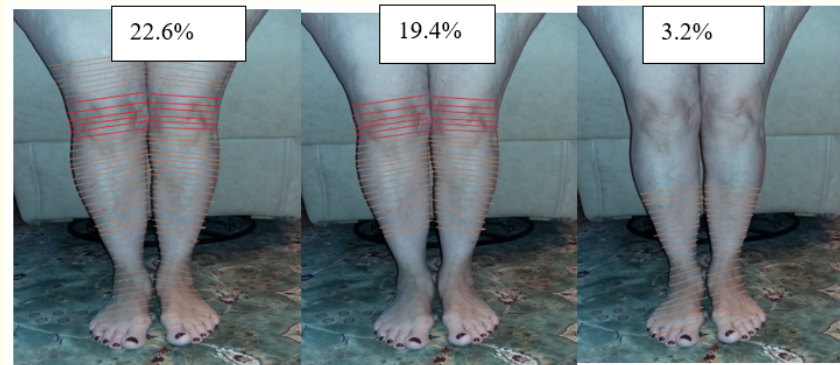

Figure 3: Types of referred hyperalgesia location.

Some patients also had allodynia (pain on brush touch). Allodynia in patients with DN4 $\geq 4$ occurred in $18.8 \%$ vs $19.2 \%$ in patients with $\mathrm{DN} 4<4,(\mathrm{p}=0.957)$.

Comparison groups in accordance with referred hyperalgesia presence.

As secondary hyperalgesia considers being a clinical feature of central sensitization, the patients were divided in two groups in accordance with the presence of secondary hyperalgesia. The first group - patients with secondary hyperalgesia included $41.5 \%$ (37), the second group - without secondary hyperalgesia included $58.5 \%$ (52) patients (Figure 4).

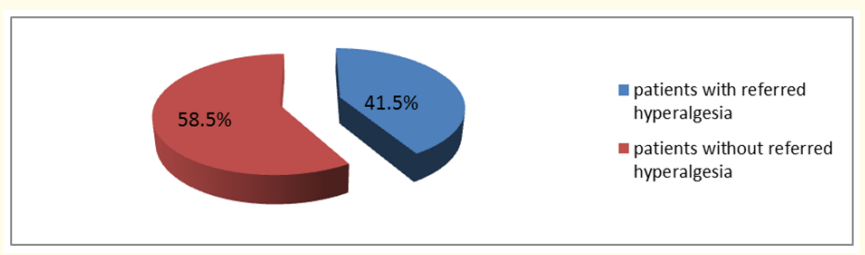

Figure 4: Dividing patients into groups in accordance to presence or absence of secondary hyperalgesia.

The statistical analysis revealed a significantly high level on the Pain DETECT scale in patients with secondary hyperalgesia $16.2 \pm$ 5.8 vs11.7 $\pm 6.5(\mathrm{p}=0.001)$ in patients without secondary hyperalgesia. In general, the level on the other neuropathic scale, DN4, was also higher in patients with secondary hyperalgesia $3.43 \pm 1.7$ vs $2.7 \pm 1.8$ ( $p=0.07$ ), but no significant difference was found.

The group with secondary hyperalgesia can be characterized by more pronounced pain on VAS $-5.9 \pm 1.5 \mathrm{~cm}(\mathrm{p}=0.013)$, a significantly higher level of depression $-8.9 \pm 2.9(\mathrm{p}=0.003)$, and more pronounced disturbance of joint function (WOMAC $1184 \pm 365(\mathrm{p}$ 
$=0.009)$ ). Examination of mood also revealed an increased level of anxiety in patients with secondary hyperalgesia $-8.9 \pm 2.9$, but no significant differences were recorded $(p=0.082)$.

Differences between groups as far as age, duration of disease, BMI or quality of life were negligible.

Both groups had predominantly Kellgren-Lawrence grade II: $69.2 \%$ patients with secondary hyperalgesia and $79.4 \%$ without secondary hyperalgesia. Almost equally frequently the patients from both groups had Kellgren-Lawrence grade III. Very rarely the patients with secondary hyperalgesia had Kellgren-Lawrence grades I and IV (Table 6).

\begin{tabular}{|c|c|c|c|}
\hline $\begin{array}{l}\text { Somatosensory } \\
\text { profile }\end{array}$ & $\begin{array}{c}\text { DN4 } \geq 4 \\
n=33\end{array}$ & $\begin{array}{c}\text { DN4<4, } \\
n=56\end{array}$ & $\mathrm{P}$ \\
\hline Sensory abnormalities & $16(76.2 \%)$ & $19(50 \%)$ & 0.046 \\
\hline Numbness & $5(16.1 \%)$ & $2(3.9 \%)$ & 0.055 \\
\hline Hyperalgesia & 17 (51.5\%) & $27(49.1 \%)$ & 0.826 \\
\hline Referred hyperalgesia & $17(58.6 \%)$ & $20(45.5 \%)$ & 0.270 \\
\hline Allodynia & $6(18.8 \%)$ & $10(19.2 \%)$ & 0.957 \\
\hline
\end{tabular}

Table 4: Somatosensory profile in patients with DN4 $\geq 4$ and DN $4<4$.

\begin{tabular}{|l|c|c|c|}
\hline Parameters & $\begin{array}{c}\text { Patients with } \\
\text { secondary } \\
\text { hyperalgesia, } \\
\mathbf{n}=\mathbf{3 7} \\
\text { Value } \pm \text { SD }\end{array}$ & $\begin{array}{c}\text { Patients without } \\
\text { secondary } \\
\text { hyperalgesia, } \\
\mathbf{n}=\mathbf{5 2} \\
\text { Value } \pm \text { SD }\end{array}$ & $\mathrm{P}$ \\
\hline Age, years & $58.58 \pm 5.7$ & $58.7 \pm 2.3$ & 0.880 \\
\hline $\begin{array}{l}\text { Duration of } \\
\text { disease, months }\end{array}$ & $121 \pm 89$ & $103 \pm 69$ & 0.279 \\
\hline Pain DETECT & $16.2 \pm 5.8$ & $11.7 \pm 6.5$ & 0.001 \\
\hline DN4 & $3.4 \pm 1.7$ & $2.7 \pm 1.8$ & 0.07 \\
\hline VAS, cm & $5.9 \pm 1.5$ & $4.8 \pm 2.3$ & 0.013 \\
\hline HADs anxiety & $9.6 \pm 3.5$ & $8.9 \pm 2.9$ & 0.082 \\
\hline HADs depression & $8.9 \pm 2.9$ & $6.9 \pm 3.4$ & 0.003 \\
\hline $\begin{array}{l}\text { EQ5D(quality of } \\
\text { life) }\end{array}$ & $0.42 \pm 0.23$ & $0.44 \pm 0.26$ & 0.773 \\
\hline WOMAC, mm & $1184 \pm 365$ & $945 \pm 469$ & 0.009 \\
\hline BMI, kg/m ${ }^{2}$ & $33 \pm 6.9$ & $34.37 \pm 7$ & 0.392 \\
\hline
\end{tabular}

Table 5: Demographic, clinical characteristics of participants.

\begin{tabular}{|l|c|c|c|}
\hline $\begin{array}{l}\text { Kellgren-Lawrence } \\
\text { grade }\end{array}$ & $\begin{array}{c}\text { Patients with } \\
\text { secondary } \\
\text { hyperalgesia, } \\
\mathbf{n = 2 6}\end{array}$ & $\begin{array}{c}\text { Patients } \\
\text { without } \\
\text { secondary } \\
\text { hyperalgesia, } \\
\mathbf{n = 3 4}\end{array}$ & P \\
\cline { 1 - 3 } I & $2(7.7 \%)$ & -- & \multirow{2}{*}{0.155} \\
\cline { 1 - 3 } II & $18(69.2 \%)$ & $27(79.4 \%)$ & \\
\cline { 1 - 3 } III & $5(19.2 \%)$ & $7(20.6 \%)$ & \\
\cline { 1 - 3 } IV & $1(1.7 \%)$ & -- & \\
\hline
\end{tabular}

Table 6: X-ray characteristics of participants.

The groups did not show differences on US data. The presence of free liquid, enlargement of synovium and signs of synovitis occurred equally frequently. Osteophytes in patients with secondary hyperalgesia occurred more often than in patients without secondary hyperalgesia $-96.8 \%$ vs $83.3 \%$, but no significant difference was found $(\mathrm{p}=0.058)$ (Table 7).

\begin{tabular}{|l|c|c|c|}
\hline US parameters & $\begin{array}{c}\text { Patients with } \\
\text { secondary } \\
\text { hyperalgesia, } \\
\mathbf{n = 3 1}\end{array}$ & $\begin{array}{c}\text { Patients without } \\
\text { secondary } \\
\text { hyperalgesia, } \\
\mathbf{n = 3 6}\end{array}$ & $\mathbf{P}$ \\
\hline Presence of liquid & $21(67.7 \%)$ & $22(61.1 \%)$ & 0.572 \\
\hline Synovium>3 мM & $21(67.7 \%)$ & $23(63.9 \%)$ & 0.740 \\
\hline Osteophytes & $30(96.8 \%)$ & $30(83.3 \%)$ & 0.058 \\
\hline Tenosynovitis & $26(83.9 \%)$ & $29(80.6 \%)$ & 0.724 \\
\hline
\end{tabular}

Table 7: USE characteristics of participants.

\section{Discussion}

The goal of our study was to explore the role of neurogenic mechanisms in chronic pain syndrome. Our data demonstrated that chronic pain in knee $\mathrm{OA}$ is a mixture of different pathogenic mechanisms. In $37 \%$ of patients, the neuropathic scale DN4 was positive. The most frequent neuropathic descriptors were burning, pins and needles, itching, electric shock-like sensation, and even numbness, tingling and increased sensitivity to cold, warmth and even touch.

The above-mentioned descriptors are the components of specified neuropathic validated scales that identify neuropathic pain. 
But clinical neuropathic pain syndrome demands presence of somatosensory nervous system damage that can be revealed by clinical and instrumental examination [18].

Although $1 / 3$ of patients with $\mathrm{OA}$ had positive results according to neuropathic DN4 and Pain DETECT scales, our neurological clinical examination did not reveal any somatosensory deficit. This is why we cannot name this pain neuropathic.

According to the last classification of chronic pain, there are 3 types of pain: nociceptive, neuropathic and dysfunctional pain, caused by central sensitization. Plasticity of the nervous system is a key mechanism of dysfunctional pain. The above-mentioned mechanisms lead to central pain neurons sensitization and pain stimulation. The core of the pathogenic pain mechanism is functional changes in the central nervous system participating in pain control. Some authors call this phenomena «augmented central pain processing» or "disturbance of sensory processioning information" [19].

The main difference between dysfunctional pain and other types is the inability to reveal the cause of pain or any organic pathology explaining the pain.

The main factors leading to dysfunctional (chronic) pain development are not organic pathology or distraction, but functional changes in the pain system and also psychological, social and emotional stress. They are the key mechanisms that alter the proper functioning of the descending noradrenergic and serotoninergic system. As a result, the patient experiences normal painless stimuli as pain [19].

Hence in the pathogenesis of chronic pain in knee OA, two mechanisms take place: nociceptive and central sensitization, and in $1 / 3$ of patients central sensitization is predominant and determines the neuropathic pattern of pain.

Central sensitization is characterized by the absence of neurological deficit and absence of pain intensity associated with structural changes in a joint. Its appearance can be explained by different neurochemical changes in CNS.

"Increased" central sensitization can be clinically investigated with the presence of neuropathic phenomena, enlargement of the pain region and secondary hyperalgesia [6]. Our study demon- strated a predominance of neuropathic pain phenomena in $37 \%$ of patients, who received more than 4 points on DN4. Secondary hyperalgesia was revealed in $41 \%$ patients with OA.

Comparative analysis between focus groups demonstrated an association between secondary hyperalgesia and neuropathic scales: Pain DETECT $(16.2 \pm 5.8$ vs. $11.7 \pm 6.5, \mathrm{p}=0.001)$ and DN4 (3.43 \pm 1.7 vs $2.7 \pm 1.8, p=0.07)$. General Practitioners and Rheumatologists can identify central sensitization with the help of routine methods: examination of sensitive sphere (revealing secondary hyperalgesia) and using neuropathic scales. For example, neuropathic scale DN4 and examination of pain sensitivity with a needle can be used to reveal secondary hyperalgesia.

Examination of the sensitive sphere with a needle revealed hyperalgesia in broad regions - hip and shin which represents an increase in the receptor field of spinal neurons and presence of central sensitization. Central sensitization plays a leading role in pathogenesis of chronic pain syndrome.

The presence of "increased" central sensitization certifies signs of neuropathic pain and secondary hyperalgesia that spread below the damaged joint.

Our findings have demonstrated that chronic pain occurs due to complex mechanisms. Besides a nociceptive mechanism, central sensitization takes place, developing due to reorganization in the pain-antipain system at the neurochemical level. In some patients, central sensitization plays a predominant role in pathogenesis of chronic pain syndrome and allows for numerous neuropathic descriptors and secondary hyperalgesia.

The presence of increased central sensitization is revealed by DN4, accompanied by more frequent observation of pins, electric shock-like sensation, tingling, numbness and itch. Induced sensations were detected more often: hyperpathia (increased sensitivity to touch and pressure), hyperalgesia (decreased pain threshold) or allodynia (pain response to painless stimuli).

Comparative analyses between groups, based on presence or absence of secondary hyperalgesia, have demonstrated an association between secondary hyperalgesia and high level of neuropathic scales, high intensity of pain on VAS, depression and high level of the functional index, WOMAC. Secondary hyperalgesia was not correlated with age, duration of disease, BMI or quality of life. 
X-ray and US examination of damaged knee data did not demonstrate a correlation between structural changes and secondary hyperalgesia.

When the pain in the knee transforms into chronic pain: a psychological component comes to the forefront in supporting pain behavior and suffering. These results can be explained by the activation of nociceptive neurons and certain supraspinal structures: the anterior cingulate cortex, the right dorsolateral prefrontal cortex, the left middle frontal gyrus, and the left lateral occipital cortex, PAG region, basal, revealed during functional MRI. The main functions of these structures are to regulate cognition, emotional processes, and behavior. These interactions provide inseparable affinity between nociception and patients behavior, including mood changes when chronic pain develops [17].

In our study, patients with $\mathrm{OA}$ did not report the presence of problems in the emotional sphere. But on psychological examination, various levels of anxiety and depression were revealed.

Mood disorders occur more often in patients with secondary hyperalgesia compared to patients who had not secondary hyperalgesia. There was a significant difference in depressive disorder.

Patients with secondary hyperalgesia did not have a significant association with anxiety, which demonstrates its value in remodeling the pain system and supporting pain in $\mathrm{OA}$, but does not play a leading role in forming central sensitization. Depression does occur significantly more often in patients with secondary hyperalgesia and this is a testament to the significant influence of depression on mechanisms of chronic pain in $\mathrm{OA}$.

The current study demonstrated structural and inflammatory processes in the knee in all patients, but a predominance and increase of clinical features of central sensitization (neuropathic descriptors and secondary hyperalgesia) in only $40 \%$ of patients. Central sensitization is associated with more intensive pain on the VAS, high level of functional activity and high level of depression.

Therefore, one of the main ways of controlling chronic pain should also target CNS mechanisms that influence its neuroplastic changes accompanied by increased excitability of spinal neurons. This is why using antidepressants, anticonvulsants (pregabalin and gabapentin) and flupertin is reasonable.
Successful treatment should also consider the role of biological, psychological and social factors in pathogenetic treatment of chronic pain and demands multidisciplinary approach in chronic pain management in specialized centers [20].

\section{Conclusion}

Our findings demonstrated that in the pathogenesis of chronic pain in knee OA, two mechanisms take place: nociceptive and central sensitization. In one-third of patients, central sensitization is predominant and determines the neuropathic pattern of pain. CS is characterized by the absence of neurological deficit and absence of pain intensity associated with structural changes in a joint. Therefore, one of the main ways of controlling pain should also target CNS mechanisms, including anticonvulsant, and antidepressant agents.

\section{Acknowledgements}

We arouse great gratitude towards Brenda S. Vollers-King, 'The Netherlands, who helped us a lot with language editing.

\section{Disclosure Statement}

The authors have declared no conflicts of interest.

\section{Bibliography}

1. Roy D Altman., et al. "Management of Ostearthritis. Knee Pain: The state of the science". Medical Education Resourses (2006).

2. Nidhi Sofat., et al. "What makes osteoarthritis painful? The evidence for Local and Central pain processing". Rheumatology 50.12 (2011): 2157-2165.

3. Doherty M. "Pain in osteoarthritis". In: Giamberardino MA, editor. Pain; 2002: an updated review: refresher course syllabus. Seattle: IASP Press (2002): 51-57.

4. Breivik H., et al. "Epub ahead of print". European Journal of Pain (2005).

5. Bjordal JM., et al. "Short-term efficacy of pharmacotherapeutic interventions in osteoarthritis knee pain: a meta-analysis of randomized placebo-controlled trials". BMJ 329 (2004): 1317.

6. NeameR., et al. "A historic issue of the Annals: three papers examine paracetamol in osteoarthritis". Annals of the Rheumatic Diseases 63 (2004): 897-900.

7. Vikki Wylde., et al. "Persistant pain after joint replacement". Prevalence, sensory qualities, and postoperative determinants". Pain 152.3 (2011): 566-572. 
8. Schaible HG., et al. "Mechanisms of pain in arthritis". Annals of the New York Academy of Sciences 966 (2002): 343-354.

9. KonttinenYT., et al. "Peripheral and spinal neural mechanisms in arthritis, with particular reference to treatment of inflammation and pain". Arthritis and Rheumatology 37 (1994): 965-982.

10. Stephen E Gwilym., et al. "Psychophysical and Functional Imaging Evidence Supporting the presence of Central Sensitization in a Cohort of Osteoarthritis Patients". Arthritis and Rheumatism 61.9 (2009). 1226-1234.

11. Jacqueline R Hochman., et al. "The nerve of Osteoarthritis Pain". Arthritis care and research 62.7 (2010): 1019-1023.

12. JR Hochman., et al. "Hawker Neuropathic pain symptoms on the modified painDETECT correlate with signs of central sensitization in knee osteoarthritis". Osteoarthritis and Cartilage 21 (2013): 1236-1242.

13. Felson DT. "The sources of pain in knee osteoarthritis". Current Opinion in Rheumatology 17 (2005): 624-628.

14. Kosek E and Ordeberg G. "Abnormalities of somato sensory perception in patients with painful osteoarthritis normalize following successful treatment". European Journal of Pain 4 (2000): 229-238.

15. Kidd BL., et al. "Arthritis and pain: current approaches in the treatment of arthritic pain". Arthritis Research and Therapy 9 (2007): 214.

16. Dieppe PA., et al. "Quantitative sensory testing in osteoarthritis of the knee [abstract]". Osteoarthritis Cartilage 16 (2008): S204.

17. Woolf CJ. "Central sensitization: implications for the diagnosis and treatment of pain". Pain 152 (2011): S2eS15.

18. Snaith RP. "The Hospital Anxiety and Depression Scale". Health Qual Life Outcomes 1 (2013): 29.

19. Bouhassira D., et al. "Comparison of pain syndromes associated with nervous or somatic lesions and development of a new neuropathic pain diagnostic questionnaire (DN4)". Pain. 114.1-2 (2005): 29-36.

20. Freynhagen R., et al. "Pain DETECT: a new screening questionnaire to identify neuropathic components in patients with back pain". Current Medical Research and Opinion 22 (2006): 1911-1920.
21. Bennett MI., et al. "Using screening tools to identify neuropathic pain". Pain 127 (2007): 199-203.

22. Treede RD., et al. "Peripheral and central mechanisms of cutaneous hyperalgesia”. Prog Neurobiol 38 (1992): 397-421.

23. Pinto M., et al. "Neuronal activation at the spinal cord and medullary pain control centers after joint stimulation: a c-fos study in acute and chronic articular inflammation". Neuroscience 147 (2007): 1076-1089.

24. Sharif Naeini R., et al. "Remodelling of spinal nociceptive mechanisms in an animal model of monoarthritis". European Journal of Neuroscience 22 (2005): 2005-2015.

25. Schaible HG., et al. "Pathophysiology and treatment of pain in joint disease". Advanced Drug Delivery Reviews 58 (2006): 323-324.

26. Yakhno NN and Kukuskin ML. "Chronic pain: medica-biological and sotsio-economic aspects". Vestnik Rossiiskoi akademii meditsinskikh nauk. Annals of the Russian Academy of Medical Sciences 9 (2012): 54-58.

27. Treede RD., et al. "Neuropathic pain: redefinition and a grading system for clinical and research purposes". Neurology 70.18 (2008): 1630-1635.

28. Danilov AB and Danilov A B. "Physician's guidance". Pain control. Biopsychosocial approach. Moscow: AMM PRESS 27 (2012): 46-85.

29. Yakhno NN and Kukuskin ML. "Chronic pain treatment: multidisciplinary approach alternative to symptomatic treatment". Russian Pain Journal 1.38 (2003): 82.

\section{Assets from publication with us}

- Prompt Acknowledgement after receiving the article

- Thorough Double blinded peer review

- Rapid Publication

- Issue of Publication Certificate

- High visibility of your Published work

Website: www.actascientific.com/

Submit Article: www.actascientific.com/submission.php

Email us: editor@actascientific.com

Contact us: +919182824667 